\title{
Efectividad del procedimiento de Cox-maze III a largo plazo su relación con el tamaño posoperatorio de la aurícula izquierda
}

Ovidio Alberto García-Villarreal*

Instituto Mexicano del Seguro Social, Hospital de Cardiología 34, Departamento de Cirugía Cardiaca, Nuevo León, México

\section{Resumen}

Introducción: El procedimiento de Cox-maze III está diseñado para eliminar la fibrilación auricular. Objetivo: Determinar la relación del tamaño posoperatorio de aurículas izquierdas en las que se realizó procedimiento de Cox-maze III. Método: De julio de 2012 a abril de 2016 se operó a 50 pacientes con enfermedad mitral primaria y fibrilación auricular concomitante. Se utilizó Cox-maze III mediante "corte y sutura" biatrial completo. La duración preoperatoria de la fibrilación auricular fue de $3.5 \pm 2.1$ años. Resultados: No hubo mortalidad operatoria. La ausencia de fibrilación auricular fue de 92,88 y $73.7 \%$ attres meses, uno y tres años. Se encontró́ relación directa entre el tamaño posoperatorio de la aurícula izquierda y la probabilidad de falla del Cox-maze cuando la aurícula izquierda fue $>6.5 \mathrm{~cm}$ a un año $(R R=10.5, I C 95 \%=4.30-26.67, p<0.0001)$ y a tres años $(R R=27.1$, IC $95 \%=3.87-189.86, p=0.0009)$. El tamaño de la aurícula izquierda disminuyó de $7.1 \pm 0.5 \mathrm{~cm}$ a $6.2 \pm 0.5 \mathrm{~cm}(p<0.0001)$. Conclusiones: El Cox-maze III es eficaz para eliminar la fibrilación auricular asociada coñ enfermedad mitral cuando el tamaño posoperatorio de la aurícula izquierda es $\leq 6.5 \mathrm{~cm}$.

PAlabRas CLAVE: Aurícula. Arritmia. Fibrilación auricular. Procedimiento de maze. Válvula mitral.

\section{Abstract}

Introduction: The cox-maze III procedure is designed to eliminate atrial fibrillation (AF). Objective: To determine the relationship of left atrial (LA) postoperative size after undergoing the Cox-maze III procedure. Method: From July 2012 to April 2016 , 50 patients with primary mitral valve disease and concomitant AF were operated on. A "cut-and-sew" cox-maze III proce-dure with a full biatrial lesion pattern was used. AF preoperative duration was $3.5 \pm 2.1$ years. Results: There was no operative mortality. Freedom from $A F$ was $92 \%, 88 \%$ and $73.7 \%$ at three months and at one and 3 years, respectively. A direct relationship was found between LA postoperative size and the probability of cox-maze failure when LA is $>6.5 \mathrm{~cm}$ at one year (relative risk $[R R]=10.5 ; 95 \%$ confidence interval $[\mathrm{Cl}]: 4.30-26.67, p<0.0001)$ and at 3 years $(R R=27.1 ; 95 \% \mathrm{Cl}: 3.87-189.86$, $p=0.0009)$. LA size decreased from $7.1 \pm 0.5 \mathrm{~cm}$ to $6.2 \pm 0.5 \mathrm{~cm}(p<0.0001)$. Conclusions: The cox-maze III procedưre is efficacious for eliminating mitral valve disease-associated $A F$ when $L A$ postoperative size is $\leq 6.5 \mathrm{~cm}$.

KEY WORDS: Atrium. Arrhythmia. Atrial fibrillation. Maze procedure. Mitral valve.

Correspondencia:

*Ovidio Alberto García-Villarreal

E-mail: ovidiocardiotor@gmail.com
Fecha de recepción: 07-07-2019

Fecha de aceptación: 14-07-2019

DOI: 10.24875/GMM.19005385
Gac Med Mex. 2019;155:590=595 Disponible en Pǘmed www.gacetamedicademexico.com 


\section{Introducción}

El procedimiento de Cox-maze ha sido diseñado para eliminar la fibrilación auricular (FA) o flutter. Está basado en el concepto de crear un laberinto (maze) o camino para el impulso eléctrico, desde el nodo sinusal hasta alcanzar el nodo auriculoventricular, pasando al través de todo el miocardio auricular. ${ }^{1}$ Con el tiempo, las incisiones quirúrgicas para crear el maze fueron sustituidas por líneas de quemadura utilizando energía alternativa; la radiofrecuencia bipolar y la crioablación son las únicas de eficacia incuestionable para lograr lesiones transmurales definitivas sobre el tejido auricular. ${ }^{2}$ Como regla general, entre más completo el patrón de lesiones del maze (mayor el número de incisiones o líneas de quemadura), mayor la tasa de éxito para libertad de $\mathrm{FA}^{3}{ }^{3}$

La complejidad del Cox-maze ha sido graduada en 9.5 y su adaptabilidad en 0.5 , ambas en una escala de 0 a 10, lo que significa que si bien es una operación segura (mortalidad operatoria $<1 \%$ ) y efectiva (> $90 \%$ ), ha tenido poco impacto en el número total de pacientes tratados. ${ }^{1}$ Por lo anterior, actualmente el Cox-maze se realiza fundamentalmente en la modalidad IV, más fácil, rápida y segura. ${ }^{4}$ No obstante, Coxmaze III continúa siendo superior a Cox-maze IV en cuanto a FA o cualquier otra taquiarritmia auricular. ${ }^{5}$

El objetivo de este artículo es presentar los resultados a largo plazo del procedimiento de Cox-maze III mediante "corte-y-sutura" para eliminar la FA en una serie de pacientes con enfermedad mitral reumática concomitante. Al mismo tiempo, se ha determinado la relación entre la recurrencia de FA después del Cox-maze y el tamaño posoperatorio de la aurícula izquierda (AI).

\section{Método}

Estudio ambispectivo en el cual se analiza el total de pacientes operados en un centro hospitalario. El periodo de estudio estuvo comprendido entre julio de 2012 y abril de 2016. Se operaron 50 pacientes, los cuales fueron sometidos a una cirugía primaria mitral y a un procedimiento de Cox-maze III en forma concomitante. Todos los pacientes firmaron la forma de consentimiento informado respectiva antes de la cirugía. Las características demográficas preoperatorias se muestran en la Tabla 1.

Los datos fueron recolectados de los expedientes clínicos y de las revisiones de los pacientes en la
Tabla 1. Variables demográficas preoperatorias en $\mathbf{5 0}$ pacientes en quienes se realizó Cox-maze III (corte-y-sutura)

\begin{tabular}{|c|c|c|}
\hline Variable & Median: & 玉正目 \\
\hline Edad (años) & $56.5 \pm$ & 8.3 \\
\hline Duración preoperatoria de la FA (años) & $3.5 \pm$ & \\
\hline Tamaño preoperatorio de la FA $(\mathrm{cm})^{\star}$ & $7.1 \pm$ & $0(5)$ \\
\hline Stroke preoperatorio & $6(12$ & $\%$ \\
\hline Grado de insuficiencia tricuspídea & $1.4 \pm$ & 1.8 \\
\hline Presión sistólica arterial pulmonar (mm Hg) & $53.8=$ & 14 \\
\hline \multirow[t]{2}{*}{ Fracción de eyección del ventrículo izquierdo (\%) } & $50.1 \pm$ & 5.4 \\
\hline & $\mathrm{n}$ & $\%$ \\
\hline Sexo (femenino) & 34 & 68 \\
\hline Etiología & & \\
\hline Reumática & 44 & 88 \\
\hline Degenerativa & 06 & 92 \\
\hline Tipo de lesión mitral & & ע \\
\hline Doble lesión mitral (estenosis e insuficiencia) & 44 & 88 \\
\hline Insuficiencia mitral pura & 6 & $\frac{72}{0}$ \\
\hline Otros diagnósticos simultáneos & & 울 \\
\hline Insuficiencia tricúspidea severa & 6 & 4 \\
\hline Doble lesión tricúspidea & 1 & $\bar{\Sigma}$ \\
\hline Doble lesión aórtica & 1 & $\triangleq$ \\
\hline Cardiopatía isquémica & 1 & $\cdot \frac{\sqrt{3}}{2}$ \\
\hline FA concomitante & 50 & 900 \\
\hline $\begin{array}{l}\text { *Diámetro mayor medido por ecocardiografía transtorácica. } \\
D E=\text { desviación estándar, } F A \text { = fibrilación auricular. }\end{array}$ & & $\begin{array}{l}\overline{0} \\
\frac{\bar{\theta}}{\overline{0}} \\
\end{array}$ \\
\hline
\end{tabular}

consulta externa en diferentes tiempos del estudio. El seguimiento fue de $4.37 \pm 1.1$ años (rango de 2.6 a 6.4 años).

El éxito fue definido de acuerdo con los criterios descritos en las guías clínicas de la Heart Rhythm $\bar{S}$ ociety como ritmo sinusal sin el uso de medicamentos antiarrítmicos clase I/III o ablaciones con catéter, i idèentificado mediante electrocardiograma estándar de 12 derivaciones en el consultorio. Solamente si existía alguna evidencia de FA o cualquier otra arritmia cardiaca, el paciente era derivado para monitoreo eleçrocardiográfico con estudio Holter de 24 horas. Așýy el seguimiento para los datos de ritmo cardiaco estuvo disponible en $100 \%$ de los pacientes elegibles a tres y a seis meses de la cirugía, $94 \%$ a un año, $90 \%$ a dos años, $81.2 \%$ a tres años, $88.9 \%$ a cuatro años, $81.3 \%$ a cinco años. Hubo cinco pacientes perdidos en el seguimiento, reportados como censurados, y cuatro muertes tardías no relacionadas con el pröcedimiento primario. Todos estos pacientes se excluyeron del estudio. No fueron motivo de investigación en ēeste estudio la calidad de vida ni la función de transporte 
de la $\mathrm{Al}$, debido a la falta de datos en la recolección desde los expedientes clínicos.

Todos los pacientes fueron sometidos a un procedimiento de Cox-maze III estándar clásico mediante "corte-y-sutura", siguiendo un patrón de lesiones biauricular completo. En todos, la cirugía se realizó como parte de un procedimiento mitral en forma integral a través del abordaje clásico de cirugía cardiaca abierta por esternotomía longitudinal y en derivación cardiopulmonar. La totalidad de los 50 pacientes fueron operados utilizando la modificación mexicana para el procedimiento de Cox-maze III, descrita por García-Villarreal, ${ }^{7}$ o alguna de sus variantes para los casos de difícil acceso a la Al. ${ }^{8}$ Es importante destacar que a partir de 2015 hemos modificado el patrón de lesiones sobre la aurícula derecha: ${ }^{9}$ se utiliza solo una contralesión sobre el anillo tricúspideo en la posición que corresponde a las dos en las manecillas del reloj, a la vez que se realiza otra incisión en $\mathrm{T}$ desde la incisión transversal en la aurícula derecha, de 1.5 a $2.5 \mathrm{~cm}$ afuera del surco auriculoventricular derecho y se prolonga hasta alcanzar la punta de la orejuela derecha. En ningún caso se utilizó criolesión sobre la cara externa del seno coronario en la línea del istmo mitral, por no contar con esta fuente de energía alternativa. En todos los casos se efectuó resección completa de la orejuela izquierda, mediante corte en su base a $1.5 \mathrm{~cm}$ por encima de la superficie externa del corazón. La resección se realizó afuera del corazón y se utilizó doble sutura continua de polipropileno $3 / 0$ para cerrar el muñón. ${ }^{7,8}$

\section{Medicamentos}

\section{DESDE el QUiRófano hasta los PRIMEROS tRES MESES}

Básicamente se manejaron amiodarona o metoprolol, warfarina, furosemida y espironolactona. A menos que hubiera alguna contraindicación formal, en todos los casos se inició amiodarona: primero, inmediatamente antes del despinzado aórtico en derivación cardiopulmonar se administró un bolo de $300 \mathrm{mg}$ intravenosos diluidos y en forma lenta durante varios minutos, seguidos de una infusión intravenosa de $900 \mathrm{mg}$ para 24 horas. Al término de esta se administraron $200 \mathrm{mg}$ vía oral cada ocho horas por tres días, luego $200 \mathrm{mg}$ vía oral cada 12 horas por tres días y finalmente $200 \mathrm{mg}$ en una sola toma al día durante los primeros tres meses después de la cirugía.

En los pacientes con alergia o intolerancia a la amiodarona, se administró metoprolol vía oral a razón de
$100 \mathrm{mg}$ cada 12 horas. Ante hipotensión o bradicardia excesiva, se redujo la dosis a $50 \mathrm{mg}$ cada 12 horras. Este régimen se mantuvo por los primeros tres meses después de la cirugía. En cuanto al anticoagulante se inició $1000 \mathrm{UI}$ de heparina no fraccionada intravenōosa por hora. Posteriormente, una vez retirados los tübos de drenaje torácico, la heparina se sustituyó por warfarina, con rangos de INR entre 2.0 y 3.5 por al menos cuatro meses después de la cirugía. Además, dădo que en todos los casos se resecó la orejuela izquieŕda, se manejó doble terapia diurética a base de $20 \mathrm{mg}$ de furosemida intravenosa cada seis a ocho horas en el posoperatorio inmediato, pasando luego a $40 \mathrm{mguvía}$ oral cada 24 horas en combinación con $25 \mathrm{mg}$ desespironolactona vía oral cada 12 horas. Así se mantuvo por el primer mes, eliminando después la espironolactona y manteniendo la furosemida por los priméros tres meses posoperatorios.

\section{DESPUÉS DEL TERCER MES POSOPERATORIO}

Después de realizar la primera pesquisa para cơrroborar el ritmo cardiaco mediante estudio Holter de ritmo cardiaco de 24 horas o electrocardiograma en el consultorio al final de tercer mes posoperatorio, si el paciēnte estaba libre de FA o cualquier otra taquiarritmia auriç্টlar, se procedió a suspender el medicamento antiarrítmicico, manteniendo solo la warfarina. Al cuarto mes posoperatorio, se realizó un electrocardiograma estándar de 12 derivaciones para corroborar la ausencia de FA o flutter. Si el paciente se mantenía libre de FA o flutter, se procedió a eliminar la warfarina, en ausencia de cualquiier otra indicación formal para el uso de esta (prótesis mmecánica, Al gigante). La furosemida usualmente se imterrumpió después del cuatro mes de la operación. Noơ se incluyó en forma sistemática la terapia de cardioversión durante los primeros tres meses debido a que nò es común contar con el recurso en forma sistemática.

\section{Análisis estadístico}

Los datos continuos se presentan como media \pm desviación estándar y los datos categóricos, cø̈mo frecuencias porcentuales. El valor de $p<0.05$ ob̆tenido mediante prueba con dos colas fue considerado estadísticamente significativo. El análisis de los dagtos fue realizado con el programa XIstat (Addinsoft SARL) para Microsoft Excel ${ }^{\circledR}$. Para el análisis univariadö se utilizó la t de Student y la U de Mann-Whitney para las variables continuas; para las variables categóricas, la prueba exacta de Fisher. 
Tabla 2. Variables intraoperatorias en 50 pacientes en quienes se realizó Cox-maze III (corte-y-sutura)

\begin{tabular}{|c|c|c|}
\hline Variable & \multicolumn{2}{|c|}{ Mediana $\pm \mathrm{DE}$} \\
\hline $\begin{array}{l}\text { Estancia en unidad de cuidados intensivos } \\
\text { (días) }\end{array}$ & \multicolumn{2}{|c|}{$2.9 \pm 0.7$} \\
\hline Estancia intrahospitalaria (días) & \multicolumn{2}{|c|}{$9.3 \pm 1.9$} \\
\hline Tiempo de derivación cardiopulmonar (minutos) & \multicolumn{2}{|c|}{$142.6 \pm 21.2$} \\
\hline Tiempo de pinzamiento aórtico (minutos) & \multicolumn{2}{|c|}{$116.8 \pm 19.2$} \\
\hline & n & $\%$ \\
\hline Patrón del maze biauricular completo & 50 & 100 \\
\hline $\begin{array}{l}\text { Tipo de procedimiento mitral } \\
\text { Prótesis biológica mitral } \\
27 \mathrm{~mm} \\
29 \mathrm{~mm} \\
31 \mathrm{~mm} \\
\text { Prótesis mecánica mitral } \\
27 \mathrm{~mm} \\
29 \mathrm{~mm} \\
\text { Reparación valvular mitral } \\
\text { Resección cuadrangular + anuloplastia con } \\
\text { anillo } \\
\text { Neocuerdas + anuloplastia con anillo }\end{array}$ & $\begin{array}{l}43 \\
10 \\
23 \\
10 \\
3 \\
2 \\
1 \\
4 \\
3 \\
1\end{array}$ & 86 \\
\hline $\begin{array}{l}\text { Tipo de procedimiento concomitante secundario } \\
\text { Reparación valvular tricuspídea con anillo } \\
\text { Revascularización coronaria } \\
\text { Prótesis biológica aórtica } \\
\text { Reoperación por sangrado } \\
\text { Mortalidad operatoria } \\
\text { Trombos en aurícula izquierda } \\
\text { Trombos en orejuela izquierda }\end{array}$ & $\begin{array}{c}6 \\
1 \\
1 \\
2 \\
0 \\
6 \\
14\end{array}$ & $\begin{array}{c}4 \\
0 \\
12 \\
28\end{array}$ \\
\hline $\begin{array}{l}\text { Morfología de la orejuela izquierda } \\
\text { Cactus } \\
\text { Ala de pollo } \\
\text { Veleta } \\
\text { Coliflor }\end{array}$ & $\begin{array}{c}21 \\
17 \\
7 \\
5\end{array}$ & $\begin{array}{l}42 \\
34 \\
14 \\
10\end{array}$ \\
\hline
\end{tabular}

\section{Resultados}

No hubo mortalidad operatoria. Se reportaron cuatro muertes tardías durante el seguimiento a largo plazo, de las cuales dos fueron accidentales, una neumonía y una por falla cardiaca terminal. La incidencia de colocación de marcapaso definitivo fue de un solo caso (2\%). Las variables intraoperatorias se pueden consultar en la Tabla 2.

El análisis del ritmo cardiaco en tiempo progresivo, así como las tendencias lineares del mismo están representadas en la Figura 1. En la etapa temprana, durante la fase de permanencia intrahospitalaria, 16 pacientes (32\%) hicieron algún tipo de trastorno del ritmo supraventricular diferente al ritmo sinusal normal. De estos, solo dos ( $4 \%$ del total) permanecieron en FA en el seguimiento, uno (2\%) permaneció como bloqueo
Tabla 3. Riesgo relativo para fibrilación auricular después del Cox-maze, de acuerdo con el tamaño de la aurícula izquierda

\begin{tabular}{|c|c|c|c|c|}
\hline $\begin{array}{l}\text { Tiempo de } \\
\text { estudio } \\
\text { (años) }\end{array}$ & $\begin{array}{c}\text { Tamaño } \\
\text { aurícula } \\
\text { izquierda }(\mathrm{cm})\end{array}$ & $\begin{array}{l}\text { Riesgo } \\
\text { relativo }\end{array}$ & IC $95 \%$ & 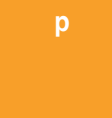 \\
\hline \multirow{2}{*}{3 meses } & $\leq 6.5$ & 0.116 & $0.0083-1.61$ & 0.1088 \\
\hline & $>6.5$ & 7.667 & $3.630-16.16$ & $<0.0001$ \\
\hline \multirow{2}{*}{1 año } & $\leq 6.5$ & 0.093 & $0.0065-1.30$ & 0.0797 \\
\hline & $>6.5$ & 10.500 & $4.130-26.67$ & $<0.0001$ \\
\hline \multirow{2}{*}{3 años } & $\leq 6.5$ & 0.1292 & $0.020-0.8089$ & 2 \\
\hline & $>6.5$ & 27.125 & $3.87-189.86$ & \\
\hline \multirow{2}{*}{5 años } & $\leq 6.5$ & 0.500 & $0.125-1.999$ & 80 \\
\hline & $>6.5$ & 12.000 & $0.634-226.99$ & 0.0 \\
\hline${ }^{*} p<0.001$ & & & & 5 \\
\hline
\end{tabular}

auriculoventricular completo y dos más (4\%) siguieron en ritmo de la unión. El resto regresó a ritmo sinusal en $8.1 \pm 1.7$ días después de la cirugía. En el seguimiênto a largo plazo, la libertad de FA estuvo presente eno92, $90,88,86$ y $73.7 \%$ a tres meses y seis meses, Gno, dos y tres años, respectivamente. A cinco años, la probabilidad de seguir en ritmo sinusal normal fue de $70 \%$. En ningún caso se realizó la aplicación de crioleșión sobre el seno coronario debido a carecer de dichörecurso en el momento comprendido por este estudie.

Eliminando los cuatro casos de mortalidad tardía y cinco más que se perdieron en el seguimiento, al último y más reciente punto de corte de este estudio, conduna mediana de 4.21 años (rango, 2.65 a 6.24 años), e[ seguimiento se cumplió completamente en 41 casos (82\%), de los cuales 30 (73.1\%) estaban libres de FA en ritmo sinusal normal y 12 casos $(24.4 \%)$ continuaban tomando algún tipo de medicamento antiarrítmico.

Al analizar la relación del tamaño posoperatoriø de la Al y la posibilidad de desarrollar FA a un plazò de tiempo variable, encontramos que el valor crítico a partir del cual se elevaba en forma alarmante dicha posibilidad para todos los cortes de tiempo fue $6.5 \mathrm{~cm}$ de diámetro mayor de la Al obtenido por ecocardiografía transtorácica (Figura 2). El riesgo relativo fue de 7.6 (IC $95 \%=3.630-16.16, p<0.0001$ ) para tres meses, 10.5 (IC $95 \%=4.130-26.67, p<0.0001$ ) para un año, 27.1 (IC $95 \%=3.87-189.86, p=0.0009$ ) para tres años y 12 (IC $95 \%=0.634-226.99, p=0.0976)$ para cinco años (Tabla 3). Lo anterior indica una clara tendencia hacia la recurrencia de la FA cuandö el diámetro posoperatorio de la Al es $>6.5 \mathrm{~cm}$. Respecto al tamaño preoperatorio y posoperatorio de lẫ Al, la asociación entre las dos medias independientes 


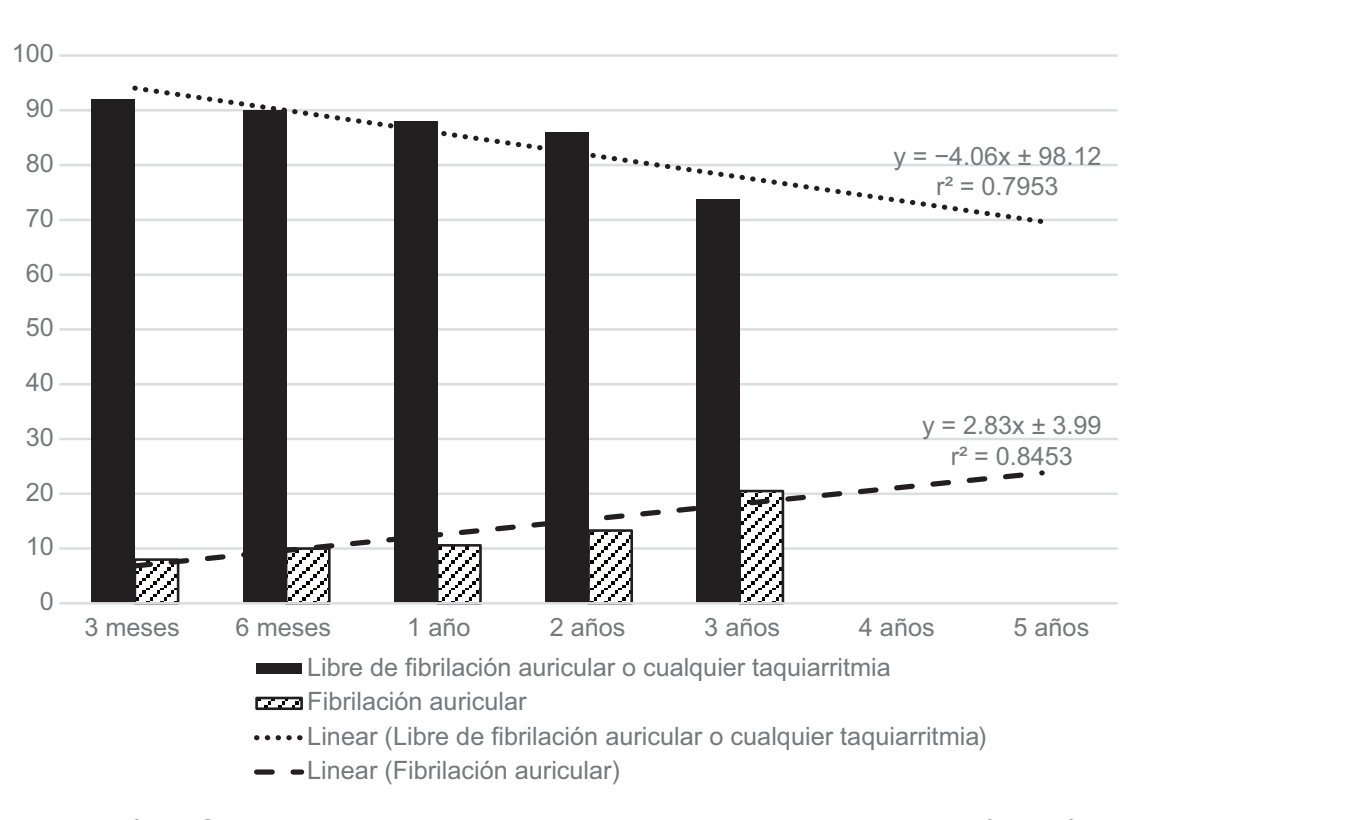

Figura 1. Ritmo cardiaco después del Cox-maze III. Las barras en color negro representan los casos libres de fibrilación o cualquier taquiarर्itmia auricular; las barras con entramado, los de fibrilación auricular. El eje vertical indica el porcentaje de los casos involucrados.

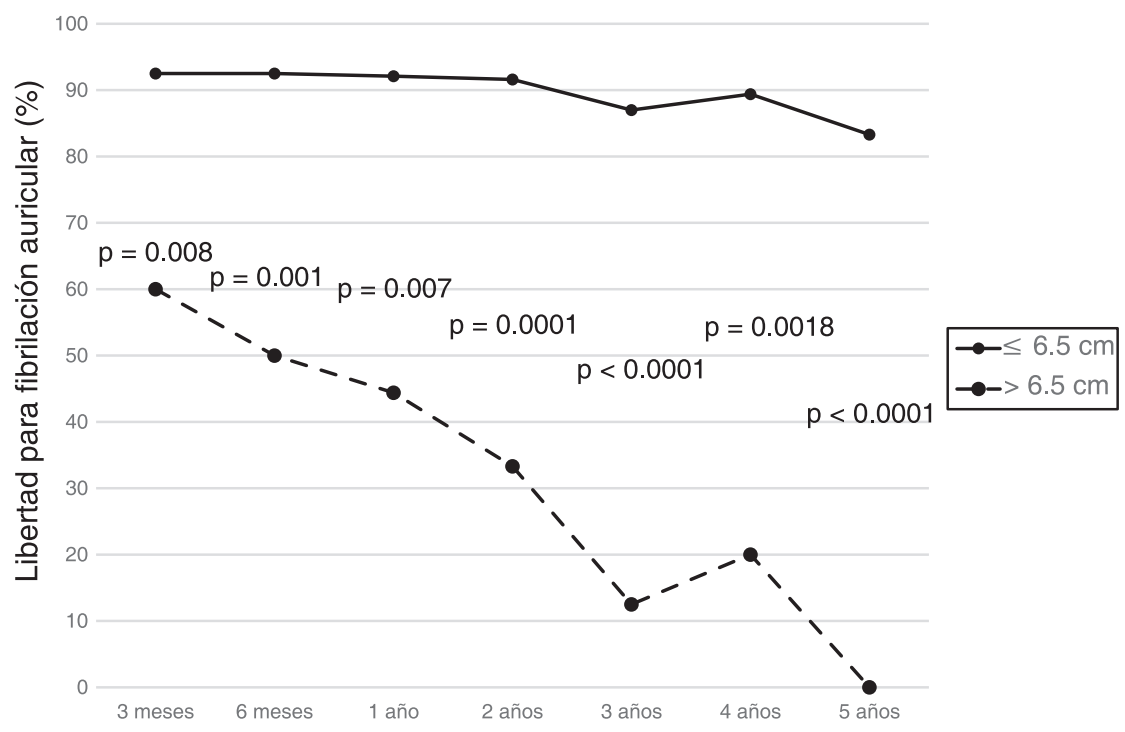

Figura 2. Ritmo cardiaco después del procedimiento de Cox-maze III, según el tamaño de la aurícula izquierda. La línea continua representa los casos libres de fibrilación auricular con un diámetro posoperatorio de la aurícula izquierda $\leq 6.5 \mathrm{~cm}$. La línea entrecortada representa los casos libres de fibrilación auricular con un diámetro posoperatorio de la aurícula izquierda $>6.5 \mathrm{~cm}$.

$(7.1 \pm 0.5$ versus $6.2 \pm 0.5 \mathrm{~cm})$ indica que el valor de $p<0.0001$ es estadísticamente significativo.

Ningún paciente presentó embolismo en ningún momento del seguimiento.

\section{Discusión}

En cuanto a la conversión a ritmo sinusal con el Cox-maze III, Cox et al..$^{10}$ describieron una tasa de efectividad superior a $90 \%$ a largo plazo. En un metaanálisis, Chen et al. ${ }^{11}$ reportaron una tasa de recuperación de ritmo sinusal de $74.6 \%$ versus $18.4 \%$ a cinco años $(p<0.0001)$ a favor de utiëzar el procedimiento de Cox-maze. En nuestra serie encontramos que los pacientes estaban libres dê $F A$ en $92,90,88,86$ y $73.7 \%$ a tres y seis meses un año, dos años y tres años respectivamente, con una línea de tendencia de probabilidad de hasta $70 \%$ a cinco años. Incluso, los resultados de $88 \%$ demüestran estar por encima del estándar mínimo recomendado de $70 \%$ para libertad de FA después del ČCoxmaze a un año de seguimiento. ${ }^{2}$ 
Como ha sido señalado por García-Villarreal ${ }^{3}$ y Ad, ${ }^{12}$ el éxito del procedimiento de Cox-maze para eliminar la FA en gran medida depende de la correcta aplicación del concepto original referente al patrón de lesiones completo y biauricular. Como regla general, entre más completo sea el patrón de lesiones del Cox-maze, mayor la proporción de éxito para eliminar la FA. ${ }^{3}$ Nosotros aplicamos el patrón original de incisiones del Cox-maze III en su modalidad biauricular completo a los 50 casos de la serie.

Hemos encontrado que existe una relación directa entre la aparición de FA en el posoperartorio y el tamaño posoperatorio de la $\mathrm{Al}>6.5 \mathrm{~cm}$ de diámetro mayor medido por ecocardiografía transtorácica. Cuando el tamaño es $>6.5 \mathrm{~cm}$, el riesgo relativo para desarrollar FA es de 7.6 y 10.5 a tres meses y un año, con un valor de $p<0.0001$ para ambos casos. Este hecho guarda estrecha relación con el argumento de la "masa crítica" necesaria para desarrollar FA. Existe una relación directa entre la cantidad de tejido auricular "continuo" disponible para fibrilar y la posibilidad de desarrollar FA. ${ }^{13}$

El procedimiento de Cox-maze III realizado mediante técnica de "corte-y-sutura" tiene la particularidad de ejercer un efecto reductivo en el tamaño total de ambas aurículas, principalmente en la Al. Al comparar los tamaños preoperatorio y posoperatorio de la $\mathrm{Al}$ en los 50 pacientes, existió reducción significativa en el tamaño final de la Al $(7.1 \pm 0.5$ versus $6.2 \pm 0.5 \mathrm{~cm}$, $p<0.0001$ ) en el periodo inmediato a la cirugía (del transoperatorio a 10 días posoperatorios).

Hasta antes de nuestro reporte, ningún estudio se había enfocado específicamente al tamaño posoperatorio de la Al como factor de riesgo para desarrollar FA después del procedimiento de Cox-maze. Nosotros encontramos que el punto de inflexión es $6.5 \mathrm{~cm}$.

La reducción auricular izquierda ${ }^{14}$ ha sido propuesta bajo la premisa de la eliminación y disminución de la masa crítica. ${ }^{13}$ Debido a la gran diversidad de técnicas para efectuar la reducción auricular izquierda, actualmente solo existe una débil evidencia a favor de la reducción auricular izquierda añadida al procedimiento de Cox-maze en pacientes con Al grande. En ningún caso efectuamos reducción auricular izquierda en esta serie de pacientes sometidos a un procedimiento de Cox-maze III mediante "corte-y-sutura". Es probable que el conjunto de las múltiples líneas de sutura sobre la Al tenga un efecto sumatorio reductivo en el tamaño final de esta estructura anatómica. En nuestra serie, la relación entre el tamaño preoperatorio y posoperatorio de la $\mathrm{Al}(7.1 \pm 0.5$ versus $6.2 \pm 0.5 \mathrm{~cm})$ tuvo un valor de $p<0.0001$. El efecto reductivo Śolo lo hemos observado en los casos de Cox-maze llileon "corte-y-sutura".

Debido a que nunca dispusimos del recurso no utilizamos crioablación sobre la cara externa del seno coronario en su intersección con la línea mitral del Cox-maze. Cox et $a l^{15}$ han descrito que la faltà de aplicación de criolesión en esta área específica puede ocasionar de 15 a $20 \%$ de falla después del Coxmaze. Es muy probable que la falta de uso de la crioablación en nuestros casos pudiera explicar la tasa de recidiva de alrededor de $24 \%$ a cinco añ̆os.

En conclusión, podemos decir que el procedimiënto de Cox-maze III mediante "corte-y-sutura" es altamente efectivo para eliminar la FA en pacientes con=enfermedad mitral concomitante, especialmente cuando el tamaño posoperatorio de la $\mathrm{Al}$ es $\leq 6.5 \mathrm{cmu}$ de diámetro.

\section{Bibliografía}

1. Cox JL. Atrial fibrillation II: rationale for surgical treatment Cardiovasc Surg. 2003;26:1693-1699

2. Ad N, Damiano RJ, Badhwar V, Calkins H, La Meir M, Nitta T, $\overline{\bar{e}}$ t al. Expert consensus guidelines: examining surgical ablation for atrial fibrillation. J Thorac Cardiovasc Surg. 2017;153:1330-1354.

3. García-Villarreal OA. Standardization in maze procedure: a step towarards a better future. J Thorac Dis. 2018;10:S3887-S3889.

4. Ruaengsri C, Schill MR, Khiabani AJ, Schuessler RB, Melby SJ, Damiano RJ. The Cox-maze IV procedure in its second decade: still the gold standard? Eur J Cardiothorac Surg. 2018;53:i19-i25.

5. Stulak JM, Suri RM, Burkhart HM, Daly RC, Dearani JA, Greason KL, et al. Surgical ablation for atrial fibrillation for two decades: are the results of new techniques equivalent to the Cox maze III procedure? J Thorac Cardiovasc Surg. 2014;147:1478-1486.

6. Calkins H, Kuck KH, Cappato R, Brugada J, Camm AJ, Chen \$A; tet al. 2012 HRS/EHRA/ECAS expert consensus statement on cathetef and surgical ablation of atrial fibrillation: recommendations for patient selection, procedural techniques, patient management and follow-up, definitions, endpoints, and research trial design. J Interv Card Electrophysiol. 2012;33:171-257.

7. CTSNet [sitio web]. García-Villarreal O. Cut and sew Cox-Maze IECProcedure with Mexican modification. EE. UU.: 2016.

8. García-Villarreal OA. Mini-partial heart autotransplantation: a different way to perform the Cox-maze III. Interact CardioVasc Thorac Surg. 2015;21:147-150.

9. Cheema FH, Younus MJ, Pasha A, Cox JL, Roberts HG. An effective modification to simplify the right atrial lesion set of the Cox-cryomaze. Ann Thorac Surg. 2013;96:330-332.

10. Cox JL, Schuessler RB, Lappas DG, Boineau JP. An 8 1/2-year Gitinical experience with surgery for atrial fibrillation. Ann Surg. 1996;224:26 ₹-273.

11. Cheng DC, Ad N, Martin J, Berglin EE, Chang BC, Doukas G, -et al. Surgical ablation for atrial fibrillation in cardiac surgery: a meta-analysis and systematic review. Innovations (Phila). 2010;5:84-96.

12. Ad N. The importance of standardization in surgical ablation for atrial fibrillation. J Thorac Cardiovasc Surg. 2016;151:399-401.

13. Garcia-Villarreal OA. eComment: left atrial reduction in atrial fibrittation surgery: the critical mass. Interact Cardiovasc Thorac Surg. 2010:10:581.

14. García-Villarreal OA, Rodríguez H, Treviño A, Gouveia AB, Argüero R Left atrial reduction and mitral valve surgery: the "functional-anatomic unit" concept. Ann Thorac Surg. 2001;71:1044-1045.

15. Cox JL, Ad N. The importance of cryoablation of the coronary sinus during the Maze procedure. Semin Thorac Cardiovasc Surg. 2000;12:20-24. 